\title{
Relación entre las prácticas tecnológicas de manejo, la producción y su asociación con las épocas del año en el sistema de doble propósito del trópico mexicano \\ Relationship between technological management practices, production and its association to the seasons of the year in the Mexican tropics dual-purpose system
}

Yuridia Bautista-Martínez ${ }^{1}$, José G. Herrera-Haro ${ }^{1}$, José Antonio Espinosa-García ${ }^{2}$, Francisco Ernesto Martínez-Castañeda ${ }^{3}$, Humberto Vaquera-Huerta ${ }^{1}$, José Ricardo Bárcena-Gamma ${ }^{1}$ y Alfonso Morales ${ }^{4}$

Palabras clave: correlaciones canónicas; análisis de correspondencia; prácticas tecnológicas de manejo; variables productivas

\section{Recepción: 16-05-17 / Aceptación: 07-08-17}

\section{Resumen}

El objetivo de este estudio fue describir y evaluar la relación entre las prácticas tecnológicas de manejo de hatos de bovinos de doble propósito y su productividad. Además de su asociación con las diferentes épocas del año. Para esto se analizaron los registros mensuales de las prácticas tecnológicas y variables productivas de treinta unidades de producción de los estados de Tabasco, Chiapas y Sinaloa durante un año. Mediante la técnica de Correlaciones Canónicas se estimó la relación entre las prácticas tecnológicas: suplementación, sanidad, prevención y control de enfermedades y manejo de praderas $(\mathrm{X})$ con variables productivas: producción de leche, porcentaje de vacas en ordeño y número de crías $(\mathrm{Y})$. El análisis de correspondencia midió la asociación del uso de las prácticas tecnológicas y la época del año. Los resultados mostraron tres correlaciones canónicas, la primera fue de 3.33 y significativa, con una variabilidad observada del $89 \%$ y las prácticas tecnológicas que mejor explican las variables productivas son la suplementación, sanidad, prevención y control de enfermedades. El análisis de correspondencia mostró que los productores de Tabasco y Chiapas varían las prácticas tecnológicas de manejo del hato de acuerdo a la época de año (lluvia, seca y transición) mientras los productores de Sinaloa las realizan durante todo el año. El uso de las prácticas tecnológicas en las unidades de producción influye en las variables productivas, principalmente en la producción de leche.

\footnotetext{
${ }^{1}$ Colegio de Postgraduados, Campus Montecillo, Estado de México. E-mail: Bautista.yuridia@colpos.mx ${ }^{2}$ Centro Nacional de Investigación en Fisiología y Mejoramiento Animal, Querétaro

${ }^{3}$ Universidad Autónoma del Estado de México

${ }^{4}$ Universidad de Wisconsin Madison, Wisconsin, EE.UU

(C) Universidad De La Salle Bajío (México)
} 
Relación entre las prácticas tecnológicas de manejo, la producción y su asociación con las épocas del año en el sistema de doble propósito del trópico mexicano

\begin{abstract}
The objective of this study was to describe and assess the relationship between technological practices of herd management of cattle of dual purpose and productivity. In addition to its association with different seasons of the year. For this, the monthly records of the productive variables and technological practices of thirty farms in the States of Chiapas, Tabasco and Sinaloa were analyzed for a year. Using the Canonical Correlations analysis, the relationship between the technological practices was estimated; supplementation, health management, prevention and control of diseases, and pasture management $(\mathrm{X})$ with productive variables; milk production, percentage of milking cows and number of offspring (Y). The correspondence analysis measured the association of the use of technological practices and the seasons of year. The results showed three canonical correlations, the first one was 3.33 and significant, with an observed variability of $89 \%$ and the technological practices that best explain the productive variables are the supplementation, health management, prevention and control of diseases. Correspondence analysis showed that farmers in Tabasco and Chiapas vary the technological management practices of the herd according to the seasons of year (rain, dry and transition) while Sinaloa farmers used them throughout the entire year, regardless of the seasons of the year. The use of technological practices in farms influences the productive variables, mainly in the milk production.
\end{abstract}

Keywords: canonical correlations; correspondence analysis; technological management practices; productivity variables. 


\section{Introducción}

Una de las actividades del medio rural más diseminadas en México es la ganadería bovina de doble propósito (DP), cuya finalidad es producir leche y carne. Este sistema basa su alimentación en el pastoreo, utiliza cruzas de Bos Taurus x Bos indicus y se desarrolla principalmente en áreas tropicales (Rojo-Rubio et al., 2009). La ganadería de DP representa una importante vía de producción de carne y leche, ya que se desarrolla en más de 48 millones de ha, con un inventario de 10, 050,886 cabezas de un total de 32, 939,529 animales productores de leche y carne a nivel nacional (SIAP, 2014).

Dada la importancia de la ganadería de DP en México, es trascendental desarrollar estrategias que permitan al productor hacer eficiente el uso de sus recursos a través de las diversas prácticas tecnológicas que realiza, tales como: alimentación, sanidad, reproducción y manejo de praderas; ya que a estas se les atribuye tener un impacto en las variables productivas, principalmente en la producción de leche y ganancias de peso. Aunque también es necesario cuantificar el grado de asociación entre estas prácticas tecnológicas y la producción (GarciaMartinez et al., 2015), y de esta forma establecer de manera cuantitativa cuales son las que tienen mayor impacto en las variables productivas.

Al considerar que la producción bovina depende de diversos factores, y con el objetivo de establecer una ponderación de los mismos, se analizaron las variables productivas y prácticas tecnológicas realizadas en unidades de producción, mediante la técnica multivariada de Correlaciones Canónicas para conocer el grado de asociación existente entre ambos grupos de variables. Esta técnica permite determinar la magnitud de las relaciones que existe entre dos grupos de variables (dependientes e independientes) a través de obtener un conjunto de ponderaciones para cada grupo, de manera que las combinaciones lineales de cada conjunto tengan una máxima correlación (Hair et al., 2006).

Además, dada la diferencia en las actividades de manejo en las unidades de producción y la frecuencia de realización de estas actividades en el transcurso del año, mediante un análisis de correspondencia múltiple que provee la información necesaria para formar un espacio matemático y poder medir similitudes o disimilitudes entre individuos, se identificaron las principales actividades que los productores realizan en diferentes épocas del año (Johnson y Whichern, 1998). 
Relación entre las prácticas tecnológicas de manejo, la producción y su asociación con las épocas del año en el sistema de doble propósito del trópico mexicano

Esta información permitirá a los asesores técnicos y productores, tomar decisiones sobre la frecuencia y el uso de las diversas prácticas tecnológicas que serán prioritarias para aumentar la producción en las unidades del sistema de doble propósito.

\section{Método}

\section{Descripción del área de estudio}

Localización. La investigación se realizó en tres regiones costeras del trópico mexicano. La Región 1 se localiza en la parte central del estado de Tabasco, con coordenadas $17^{\circ} 51^{\prime} \mathrm{N}, 93^{\circ} 23^{\prime} \mathrm{O}$ y 29 msnm, clima cálido húmedo con abundantes lluvias en verano, temperatura media anual de 26.4 ${ }^{\circ} \mathrm{C}$ y precipitación media mensual de $190.85 \mathrm{~mm}$. La región II se ubica en la zona costera de Chiapas, a $15^{\circ} 41^{\prime} 12^{\prime \prime} \mathrm{N}$ y $93^{\circ} 12^{\prime} 33^{\prime \prime} \mathrm{O}$ y altitud $57 \mathrm{msnm}$, clima cálido sub húmedo, temperatura media anual de $28{ }^{\circ} \mathrm{C}$, precipitación media mensual de $80 \mathrm{~mm}$. La Región III se localiza en el

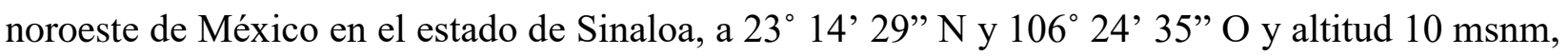
clima tropical semi húmedo, temperatura media anual de $26.0^{\circ} \mathrm{C}$, precipitación media mensual de $63 \mathrm{~mm}$ (CNA, 2016).

Manejo del ganado. El hato nacional bovino cuenta con aproximadamente 32 millones de cabezas de ganado, de las cuales el 18\% se desarrollan en las tres regiones de estudio en un sistema de doble propósito (SIAP, 2016), basado en cruzas de Bos indicus con Bos taurus, principalmente Cebú x Suizo Pardo en condiciones de pastoreo con forrajes nativos e introducidos. El tamaño de las explotaciones varía de 12 ha a 450 ha, con hatos de 40 a 200 cabezas.

Colecta de datos. Durante el periodo de julio 2012 - junio 2013 se obtuvieron datos mensuales de 30 unidades de producción (UP), diez por región, mediante encuesta directa con los productores y medición periódica de la producción y registro de eventos productivos y reproductivos en los animales. Se diseñaron cédulas para el registro y seguimiento de prácticas tecnológicas realizadas en las UP relacionadas con manejo de hato, sanidad, alimentación, manejo de praderas.

Análisis estadístico. Se realizó un análisis descriptivo de la información y se eliminaron datos aberrantes (outliers). Para estimar la asociación entre prácticas tecnológicas y variables productivas se aplicó un análisis de Correlación Canónica, usando el procedimiento PROC CANCORR de SAS 9.4 (SAS, 2013). Las prácticas tecnológicas dieron origen a la matriz X e incluyeron: $\mathrm{X}_{1}$. Uso de suplementos, $\mathrm{X}_{2}$. Manejo de praderas (MANPRA), $\mathrm{X}_{3}$. Sanidad durante la ordeña (SANORD) y $\mathrm{X}_{4}$. Prevención y control de enfermedades (PCE), cuyos valores se construyeron usando una escala 
de 1 a 4, asignando el valor de acuerdo al número de actividades realizadas. El manejo de praderas consideró la carga animal, rotación de praderas, lotificación de hato y control de malezas; la sanidad incluyó el lavado de pozones, uso de pre sellador y sellador, utilización de toallas individuales para el secado de la ubre; la prevención y control de enfermedades incluyó el diagnóstico y prevención de mastitis y brucella, aplicación de vacunas y desparasitación; el uso de suplementos alimenticios se cuantificó en meses. Las variables productivas identificaron la matriz Y, incluyó la producción promedio diaria de leche, porcentaje de vacas en ordeña y número de crías. Estas variables fueron estandarizadas para evitar sesgos debidos a la magnitud de las unidades de medida.

En forma complementaria, se usó un análisis multivariado de correspondencia múltiple usando SAS 9.4 (SAS, 2013) que permitió identificar la intensidad y estructura de la relación entre prácticas tecnológicas y época de año (lluvia, transición y seca) por región. Esta técnica permite estudiar la relación entre variables según las distancias en puntos representados gráficamente en un plano (Jhonson y Wichern, 1998). Las prácticas tecnológicas consideradas fueron: lotificación de hato (LH), ajuste de carga animal (ACA), suplementación con concentrados (SC), forrajes conservados (FC), uso de residuos agroindustriales (RA), inseminación artificial (IA), diagnóstico de gestación (DG), desparasitación interna y externa (DIYE), pre sellador y sellador (SYP), prueba de tazón de fondo oscuro (TFO), prueba de california (PC), control de malezas (CM), diagnóstico y control de brucella (DCB).

\section{Resultados}

Las prácticas tecnológicas se correlacionan positivamente con las variables productivas (Cuadro 1). La producción de leche tiene una correlación positiva con la suplementación de concentrados (0.76), manejo de praderas (0.62), prácticas sanitarias en el ordeño (0.67) y la prevención y control de enfermedades (0.63). El porcentaje de vacas en ordeño se correlacionó positivamente con la suplementación (0.73), manejo de praderas (0.55) y las prácticas sanitarias en el ordeño (0.62). 
Relación entre las prácticas tecnológicas de manejo, la producción y su asociación con las épocas del año en el sistema de doble propósito del trópico mexicano

Cuadro 1. Correlaciones simples y cruzadas de prácticas tecnológicas y variables productivas.

Características Prácticas tecnológicas Variables productivas SUPLE MANPRA SANORD PCE PLECHE PVO NCRÍAS

\begin{tabular}{llllllll}
\hline SUPLE & 1.000 & $0.532^{*}$ & $0.656^{*}$ & 0.397 & $0.760^{*}$ & $0.736^{*}$ & -0.214
\end{tabular}

MANPRA

1.00

$-0.599^{*}$

$0.623^{*}$

$0.553^{*} \quad 0.387$

0.342

SANORD

1.00

$0.49^{*}$

$0.676^{*}$

$0.623^{*}-0.090$

PCE

1.00

$0.633^{*}$

$0.350 \quad-0.276$

PLECHE

1.00

$0.747^{*}-0.402$

PVO

$1.00 \quad-0.312$

NCRÍAS

1.00

SUPLE $=$ Suplementación alimenticia; MANPRA=Manejo de praderas; SANORD=Prácticas sanitarias en el ordeño; PCE =Prevención y control de enfermedades; $\mathrm{PLECHE}=$ Producción de leche; $\mathrm{PVO}=$ Porcentaje de vacas en ordeño; NCRIAS $=$ Número de crías. ${ }^{*} \mathrm{P}<0.05$

El análisis canónico permitió identificar tres correlaciones (Cuadro 2), de las cuales la primera resultó significativa, mostrando un coeficiente de correlación de $0.87\left(\mathrm{r}^{2}=0.76\right)$ que explica el 89.0 $\%$ de la variación. La segunda y tercera correlaciones canónicas son menores y no significativas (P $>.01)$. 
Cuadro 2. Análisis de correlación de variables productivas (y) vs practicas tecnológicas (x).

\begin{tabular}{|c|c|c|c|c|c|c|c|}
\hline $\begin{array}{l}\text { Variables } \\
\text { canónicas }\end{array}$ & Combinación de variables canónicas estandarizadas & $\begin{array}{l}\text { Valores } \\
\text { propios }\end{array}$ & $\begin{array}{l}\text { Correlación } \\
\text { canónica }\end{array}$ & $\begin{array}{l}\text { Correlación } \\
\text { canónica } \\
\text { cuadrada }\end{array}$ & Proporción & Acumulado & $\begin{array}{l}\mathrm{P} \\
\text { value }\end{array}$ \\
\hline $\begin{array}{l}\text { Primer } \\
\text { conjunto } \\
\text { de } \\
\text { variables } \\
\text { canónicas }\end{array}$ & $\begin{array}{l}\mathrm{U}_{1}=0.574 * \text { SUPLE } 0.214 * \text { MANPRA+0.156*SANORD+0.295PCE } \\
\mathrm{V}_{1}=0.820 * \text { PLECHE }+0.254 * \text { PVO+0.064*NCRIAS }\end{array}$ & 3.336 & 0.877 & 0.769 & 0.890 & 0.890 & $<.0001$ \\
\hline $\begin{array}{l}\text { Segundo } \\
\text { conjunto } \\
\text { de } \\
\text { variables } \\
\text { canónicas }\end{array}$ & $\begin{array}{l}\mathrm{U}_{2}=0.366 * \text { SUPLE }+0.696 * \text { MANPRA+0.92*SANORD-0.865*PCE } \\
\mathrm{V}_{2}=-0.609 * \text { PLECHE }+1.02 * \text { PVO }+0.792 * \text { NCRIAS }\end{array}$ & 0.308 & 0.485 & 0.235 & 0.082 & 0.972 & 0.1633 \\
\hline $\begin{array}{l}\text { Tercer } \\
\text { conjunto } \\
\text { de } \\
\text { variables } \\
\text { canónicas }\end{array}$ & $\begin{array}{l}\mathrm{U}_{3}=0.398 * \text { SUPLE }+0.900 * \text { MANPRAD }+0.687 * \text { SANORD }+0.671 * \text { PCE } \\
\mathrm{V}_{3}=1.181 * \text { PLECHE- } 1.06 * \text { PVO+0.749*NCRIAS }\end{array}$ & 0.104 & 0.307 & 0.094 & 0.027 & 1.000 & 0.2885 \\
\hline
\end{tabular}

SUPLE $=$ Suplementación alimenticia; MANPRA=Manejo de praderas; SANORD=Practicas sanitarias en el ordeño; PCE =Prevención y control de enfermedades; $\mathrm{PLECHE}=$ Producción de leche; $\mathrm{PVO}=$ Porcentaje de vacas en ordeño; NCRIAS = Número de crías; $U_{1}$ a $U_{3}$ : Primera a la tercera variable canónica independiente; $\mathrm{V}_{1}$ a $\mathrm{V}_{3}$ : Primera a la tercera variable canónica dependiente.

La estructura de la correlación canónica, indica que la primera variable canónica incluye principalmente la información sobre la producción de leche $(0.820)$. Por lo tanto, la primera combinación de la variación canónica estandarizada podría ser considerada como una medida de la producción de leche predecible.

Las prácticas tecnológicas influyen en forma positiva los aspectos que integran las variables productivas (Cuadro 3). La suplementación es la que mayor peso registra en la variable canónica PRODUCTIVA1 (0.79), la segunda es la SANORD (0.707) seguido de PCE (0.590). Mientras que el manejo de praderas muestra una correlación negativa.

Cuadro 3. Correlaciones entre prácticas tecnológicas y las variables canónicas de características productivas.

\begin{tabular}{|c|c|c|c|}
\hline & $\begin{array}{l}\text { Variable canónica } \\
\text { PRODUCTIVA1 }\end{array}$ & $\begin{array}{l}\text { Variable canónica } \\
\text { PRODUCTIVA2 }\end{array}$ & $\begin{array}{l}\text { Variable canónica } \\
\text { PRODUCTIVA3 }\end{array}$ \\
\hline SUPLE & 0.797 & 0.126 & -0.049 \\
\hline MANPRA & -0.627 & 0.117 & 0.145 \\
\hline SANORD & 0.707 & 0.158 & 0.066 \\
\hline PCE & 0.590 & -0.244 & 0.166 \\
\hline
\end{tabular}


SUPLE $=$ Suplementación alimenticia; MANPRA $=$ Manejo de praderas; SANORD=Prácticas sanitarias en el ordeño; PCE = Prevención y control de enfermedades.

El análisis de correspondencia mostró una asociación entre la realización de prácticas tecnológicas y época de año en la región central de Tabasco y costera de Chipas (Figura 1). La época de seca mostró una asociación con el ajuste de carga animal (ACA), rotación de praderas (RP), desparasitación interna y externa (DIYE), suplementación con forrajes conservados (FC), suplementación con concentrados (SC), y control de malezas en las praderas (CM). Mientras que las prácticas tecnológicas asociadas a la apoca de lluvia fueron; prueba de california para la detección de mastitis (PC), uso de sellador y pre sellador en la ordeña (SYP) y la suplementación con residuos agroindustriales (RA). En la época de transición el uso del tazón de fondo oscuro para diagnóstico de mastitis (TFO).

Figura 1. Análisis de correspondencia entre prácticas tecnológicas de manejo y época del año en el estado de Tabasco y Chiapas.

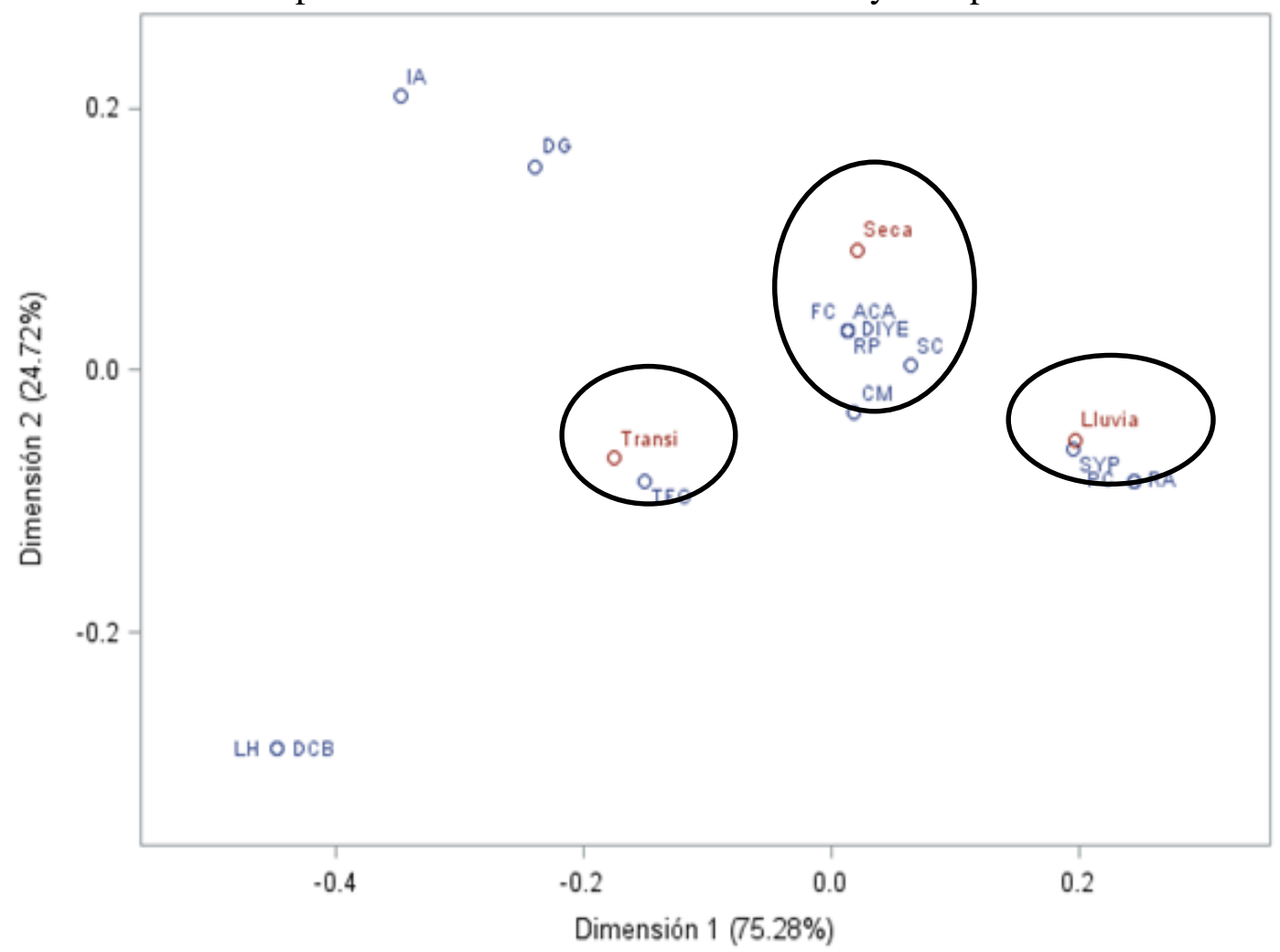

LH= Lotificación de hato; $\mathrm{ACA}=$ Ajuste de carga animal; $\mathrm{SC}=$ Suplementación con concentrados; $\mathrm{FC}=$ Forrajes conservados; $\mathrm{RA}=$ Uso de residuos agroindustriales; $\mathrm{IA}=$ Inseminación artificial; DG= Diagnóstico de gestación; DIYE= Desparasitación 
interna y externa; $\mathrm{SYP}=$ Pre sellador y sellador; TFO= Prueba de tazón de fondo oscuro; $\mathrm{PC}=$ Prueba de california; $\mathrm{CM}=$ Control de malezas; $\mathrm{DCB}=$ Diagnóstico y control de brúcella.

El análisis de correspondencia para la región noroeste del estado de Sinaloa (Figura 2), mostró que los productores realizan las siguientes practicas tecnológicas durante las tres épocas del año (lluvia, transición y seca): forrajes conservados (FC), suplementación con concentrados (SC) y de residuos agroindustriales (RA), rotación de praderas $(\mathrm{RP})$, lotificación de hato $(\mathrm{LH})$, desparasitación interna y externa (DIYE), inseminación artificial (IA), prueba de california para la detección de mastitis (PC), uso de sellador y pre sellador en la ordeña (SYP).

Figura 2. Análisis de correspondencia prácticas tecnológicas de manejo y épocas del año en la Región noroeste de Sinaloa.

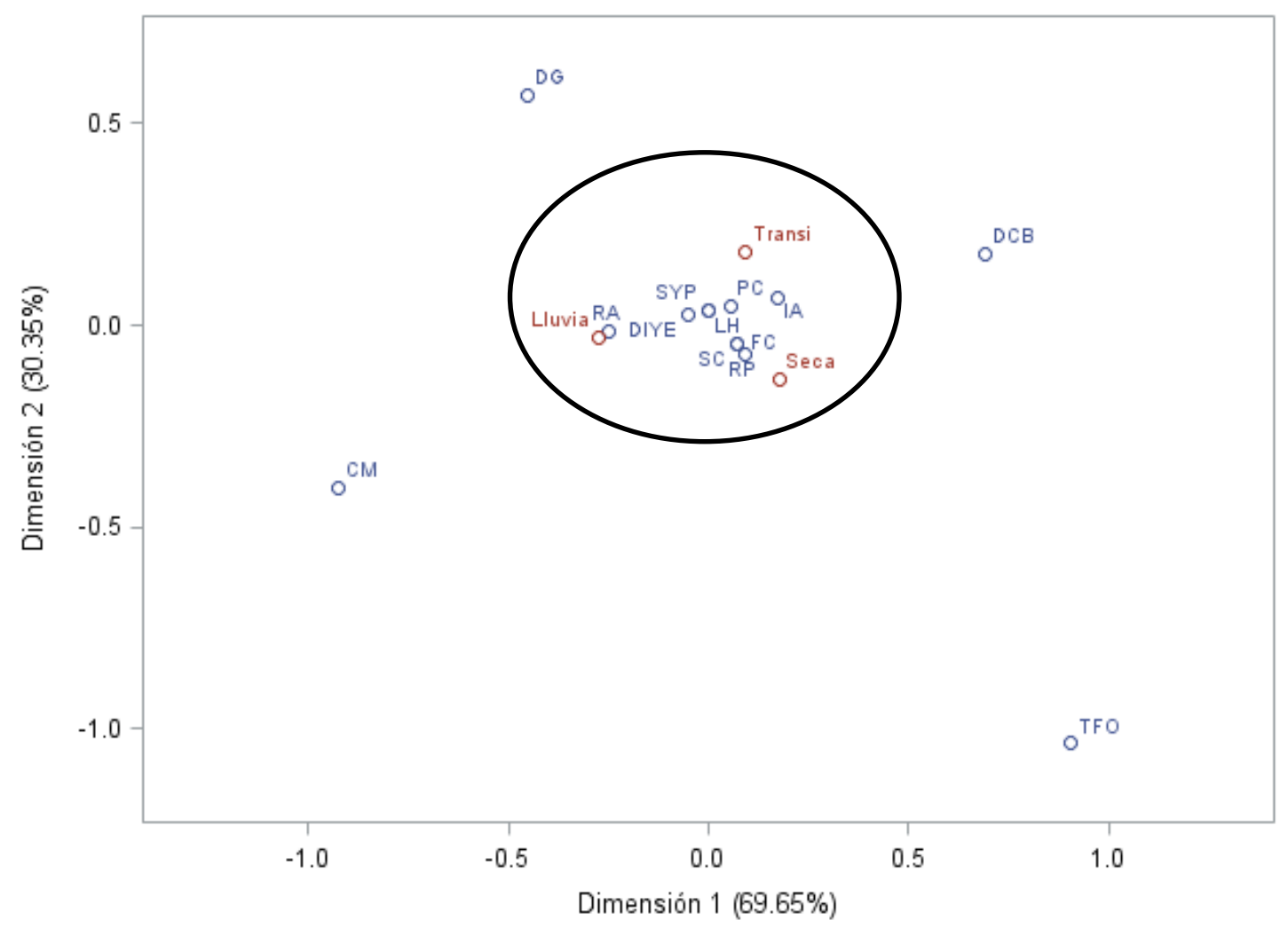

LH= Lotificación de hato; $\mathrm{ACA}=$ Ajuste de carga animal; $\mathrm{SC}=$ Suplementación con concentrados; $\mathrm{FC}=$ Forrajes conservados; $\mathrm{RA}=$ Uso de residuos agroindustriales; $\mathrm{IA}=$ Inseminación artificial; DG= Diagnóstico de gestación; DIYE= Desparasitación interna y externa; $\mathrm{SYP}=$ Pre sellador y sellador; $\mathrm{TFO}=$ Prueba de tazón de fondo oscuro; $\mathrm{PC}=$ Prueba de california; $\mathrm{CM}=$ Control de malezas; $\mathrm{DCB}=$ Diagnóstico $\mathrm{y}$ control de brúcella. 
Relación entre las prácticas tecnológicas de manejo, la producción y su asociación con las épocas del año en el sistema de doble propósito del trópico mexicano

\section{Discusión y Conclusión}

La suplementación alimenticia está correlacionada positivamente con la producción de leche. Esta correlación coincide con lo reportado por Balocchi et al., (2002) quienes suplementaron vacas en pastoreo con un concentrado a base de pulpa seca de remolacha y otro a base de granos $\left(6 \mathrm{~kg} \mathrm{vaca}^{-}\right.$

1. $\left.\mathrm{d}^{-1}\right)$. Los animales que recibieron suplemento produjeron más leche que animales que solo estaban en pastoreo, mientras que Montiel et al., (2007) encontraron que la suplementación alimenticia con concentrado (1\% del peso vivo) aumentó la producción de leche. Esto es importate, ya que la producción de forrajes en cantidad y calidad es variable en el trascurso de año, Montiel et al., (2007) reporta que en el trópico mexicano en la época de invierno hay una baja disponibilidad de forraje, y la madurez de éste en el verano, no permite satisfacer los requerimientos de las vacas en producción de leche, por lo que recomienda suplementar con concentrados en estas épocas para reducir las variaciones en la producción de leche durante el año.

Las diferentes prácticas tecnológicas relacionadas con el manejo de praderas tales como: correcta carga animal, rotación de praderas, control de malezas, fertlizacion, están relacionadas positivamente con la producción de leche y ganancia diaria de peso (Vázquez y Smith 2000). Sin embargo, un manejo inadecuado de las praderas tiene un efecto negativo en las variables de produccion, como el caso de los resultados en esté estudio sobre las variables productivas.

Realizar prácticas sanitarias durante el ordeño tiene un impacto positivo en el porcentaje de vacas en ordeño y la producción de leche. Estas prácticas reducen la incidencia de mastitis en el hato, evitando que las vacas sean desechadas y aumenten su vida productiva. (Caicedo et al., 2011).

La prevención y control oportuno de enfermedades en el hato se correlaciona positivamente con la producción de leche. Caicedo et al. (2011), mencionan que las principales razones de desecho en vacas son: mastitis y fallas reproductivas. Y el objetivo del diagnóstico y control de enfermedades es minimizar las pérdidas de productividad debido a enfermedades (Trujillo et al., 2011).

El análisis de correlaciones canónicas indica un alto grado de asociación entre las prácticas tecnológicas y las variables de producción en el sistema de producción bovino de doble propósito, ya que explica un $89 \%$ de la variación observada. Las diferentes prácticas tecnológicas en el manejo del hato son importantes, ya que de estas dependerán los resultados de la producción de carne y leche. Castro et al., (2012), mencionan que los principales problemas que impiden el 
éxito en el desarrollo de la producción lechera de doble propósito son el inadecuado manejo en la alimentación, reproducción y programas sanitarios para la prevención y el control de enfermedades, puesto que son los factores que más fuertemente repercuten en los parámetros productivos como la ganancia diaria de peso y la producción de leche.

La correlación entre las prácticas tecnológicas y las variables canónicas de características productivas, la suplementación alimenticia es la que mayor peso tiene en la variable canónica PRODUCTIVA1. La suplementación de vacas en pastoreo permite compensar los desbalances nutricionales de la pradera y aumentar en consumo de nutrientes, fundamentalmente energía, mejorando la producción de leche. Pulido et al. (2001) suplementaron con $2.7 \mathrm{~kg}$ de concentrado por día a vacas en gestación bajo un sistema de pastoreo y la producción de leche aumento de 15.1 a 20.2 litros por vaca, además se mejoró la condición corporal al parto. Una alimentación que no cubre los requerimientos nutricionales de los animales causará una baja condición corporal, y baja fertilidad.

La sanidad en el ordeño fue la variable que tuvo la segunda mayor correlación con la variable canónica PRODUCTIVA 1. Realizar prácticas sanitarias tales como, lavado de pezones, uso de pre sellador, sellador y utilización de toallas individuales para el secado de la ubre mejora las variables productivas. Bedoña y Leon, (2008) menciona que el no realizar estas prácticas provoca que el canal del pezón quede abierto y que las bacterias presentes en el medio ambiente ingresen con facilidad generando una mastitis sub-clínicas, que, si no son detectadas a tiempo, pueden convertirse en mastitis clínicas con disminución en la producción y pérdida de la función de los cuartos afectados en la ubre. Bermudez y Silvestri, (2006) mencionan que para obtener una mayor producción de leche y de buena calidad, las unidades de producción deben implementar una rutina de ordeño, ya sea la ordeña manual o mecánica, que cumpla con las siguientes reglas: ordeñar pezones limpios, desinfectados y secos, usar un equipo limpio y desinfectado, buenas prácticas de limpieza y de mantenimiento de los equipos, utensilios y elementos de ordeño. Estas prácticas sanitarias disminuyen la incidencia de mastitis en las vacas, evitando que estas sean desechadas antes de que terminen su vida productiva.

La prevención y control de enfermedades mostró un efecto sobre la variable PRODUCTIVA1. Waard y Jacobus, (2010) señalan que la implementación de un programa sanitario en las unidades de producción tiene un efecto positivo en la producción animal, ya que 
animales enfermos disminuyen la producción de leche, pierden peso gradualmente y por consiguiente hay pérdidas de rendimiento de la canal en el rastro.

Para realizar las prácticas tecnológicas de manejo del hato en unidades de producción de las regiones, centro de Tabasco y costera de Chiapas, utilizan como criterio la época del año. En estas regiones la suplementación con concentrados comerciales y forrajes conservados está asociada con la época de seca, debido a que en esta época hay una baja de disponibilidad de forraje, tanto en cantidad como en calidad, impidiendo que los animales cubran sus requerimientos nutricionales. Oviedo et al. (2011) mencionan que esta situación motiva a los productores a buscar alternativas, para reducir las pérdidas de peso y producción de leche.

El ajuste de carga animal está asociado a la época de seca. Al haber una baja disponibilidad de forraje en cantidad y calidad en esta época, es importante realizar un ajuste de carga animal, para hacer un uso eficiente de forraje disponible en las praderas (Esqueda-Esquivel et al., 2009). Diversos estudios han demostrado que un uso irracional de las praderas como el sobrepastoreo de estas, especialmente en época de seca, aumenta la cantidad de malezas en los potreros (Hernández et al., 2012). Es por ello que la época de secas es ideal para realizar control de malezas, ya que de no realizarse en está época, al inicio de la temporada de lluvias habrá una invasión de éstas, especialmente después de una sequía prolongada.

Por otra parte, la desparasitación interna y externa está asociada a la época de seca, aunque el calendario de desparasitación es dinámico y se da en el trascurso de año. Los parásitos externos como moscas y garrapatas, tienden a proliferar más en esta época, ya que las condiciones de temperatura y húmedad son idóneas para su desarrollo y hay un aumento en el uso de desparasitantes externos e internos (Mariscal y Moreno, 2013). Por otra parte, los productores comunmente hacen una desparasitación de amplio espectro a finales de época seca (Houdijk et al., 2011), con el objetivo de disminuir la cantidad de parásitos que se hospedan dentro del animal y con esto reducir la tasa de parasitosis en la próxima temporada de lluvia, ya que en esta época en climas tropicales al haber una mayor precipitación, humedad y temperaturas altas, se crea un ambiente adecuado para el desarrollo de parásitos (Torres-Acosta et al., 2006).

Los productores prefieren realizar la prueba de california para la detección de mastitis, uso de pre sellador y sellador en los pezones después de la ordeña en la época de lluvia, ya que en esta época por exeso de humedad aumenta la proliferación y transmisión de organismos patógenos, lo cual incrementa el riesgo de mastitis subclínica (Pinzón et al., 2009) en la cual el animal no 
presenta inflamación en la glándula mamaria, dolor al manipular los pezones en la ordeña, ni disminución en la producción de leche. Y la prueba de california permite identificar los cuartos de la ubre que tienen el problema y con ésto aplicar medidas de control antes que la condición del animal empeore (Pinzón et al., 2009). Mientras que la prueba del tazón de fondo oscuro para la detección de mastitis, los productores la prefieren realizar durante la época de transición, por ser más práctica y los riesgos de incidencia de mastitis en esta época en el hato son menores, aunado a que la practica preventiva del uso de toallas individuales para el secado de ubre, se realiza durante todo el año y disminuye el riesgo de infección, evitando la propagación de patógenos infecciosos (Fernández et al., 2008).

Por otra parte, los residuos agroindustriales para la alimentación son utilizados con mayor frecuencia en la época de lluvia, esto se atribuye a que en esta época existe una mayor disponibilidad de forraje y de buena calidad, algunos de estos productos son suficientes para cubrir los requerimientos de los animales, aumentar ganancias de peso y producción de leche, y así disminuir costos respecto a uso de concentrados comerciales (Sánchez et al., 2010).

Las practicas tecnológicas del manejo de hato en el noroeste de Sinaloa, se realizan de manera constante durante las tres épocas de año (lluvia, transición y seca). La suplementación con forrajes conservados, concentrados y residuos agroindustriales se realizan durante todo el año, esto puede deberse a que la precipitación promedio mínima y máxima mensual es $2 \mathrm{~mm}$ en el mes de abril y $217 \mathrm{~mm}$ en el mes de septiembre, menores respecto a Tabasco y Chiapas que presentan un promedio mínimo mensual de 64.2 y 45.3 en el mes de abril respectivamente y un promedio de máxima mensual de 356.1 y $444.3 \mathrm{~mm}$ en el mes de septiembre (CNA, 2016). En el noroeste de Sinaloa, la precipitación media mensual $(63 \mathrm{~mm})$ no es la suficiente para exista la cantidad necesaria de forraje para pastorear a los animales y cubrir sus requerimientos nutricionales necesarios. En época de lluvia cuando hay abundancia de forraje, los productores siembran y conservan estos para utilizarlos como suplementación en el transcurso del año. En el trópico subhúmedo existe una producción irregular de forrajes consecuencia de la estacionalidad de la precipitación, esto determina la escasez y baja calidad de los forrajes de los pastos durante la época de seca, provocando bajos índices productivos y reproductivos del ganado en este periodo (OrtegaJiménez et al., 2009). Para evitar que los índices productivos y reproductivos disminuyan, los productores buscan alternativas tecnológicas y de aplicación de innovaciones en el área de la 
alimentación del ganado y forrajes como prioridad, adaptando tecnologías tales como: rotación de praderas (RP) y lotificación de hato (LH), durante todas las épocas del año (Cuevas et al., 2013).

El alto grado de asociación entre el grupo de variables productivas y las diferentes actividades de manejo del hato son importantes ya que de estas dependerá en gran parte los resultados en la producción. Destacando que el uso de suplementos, sanidad y prevención y control de enfermedades influyen en las variables productivas. El poner mayor atención en las actividades de manejo que repercuten más fuertemente en las variables productivas puede tener mayores beneficios para los productores.

El análisis de correspondencia indica que los productores de la región centro de Tabasco y costa de Chiapas varían las prácticas de manejo de acuerdo a la época de año, esto es importante ya que de acuerdo a los recursos con los que cuenten las unidades de producción, se toman decisiones tales como suplementar con concentrados para cubrir los requerimientos de los animales y no disminuir la producción de leche y carne. Mientras los productores del noroeste de Sinaloa, realizan las prácticas tecnológicas de manera uniforme durante todo el año, situación que es importante dentro del sistema productivo, ya que así se asegura una producción animal menos variable.

\section{Referencias}

Balocchi, L., O.F. Pulido \& V.J. Fernández. (2002). Comportamiento de vacas lecheras en pastoreo con y sin suplementación con concentrado. Agricultura Técnica 62: 87-98.

Bedolla, C. C \& M. P. León. (2008). Pérdidas económicas ocasionadas por la mastitis bovina en la industria lechera. Revista Electrónica de Veterinaria 9: 1- 26.

Bermúdez, E. N \& J. A. S. Silvestre. (2006). Análisis del proceso de ordeño y de la calidad higiénica de la leche utilizada en la fabricación del queso Paipa en el municipio de Paipa (Boyacá), Colombia. Revista de Investigación Universidad La Salle 6: 163-170.

Caicedo, R.R., G. J. L. Garita \& N. Paz-Calderón. (2011). Salud animal de una cuenca lechera bajo el sistema de traspatio, Puebla, México. AICA 1: 323-326.

Castro, C. J. M., J. C. Rivera \& Zavaleta, J. A. (2012). Características de la producción y comercialización de leche bovina en sistemas de doble propósito en Dobladero, Veracruz. Revista Mexicana de Agronegocios 30: 816-824.

CNA. (Comisión Nacional del Agua). (2016). http://smn.cna.gob.mx (Consulta: enero 2017). 
Cuevas R. V., M. J. Baca, E.F. Cervantes, E. García, J. Antonio \& J. (2013). Factores que determinan el uso de innovaciones tecnológicas en la ganadería de doble propósito en Sinaloa, México. Revista mexicana de ciencias pecuarias 4:31-46.

Esqueda-Esquivel, V. A., M. Montero-Lagunes \& F.I. Juárez-Lagunes. (2009). Efecto de métodos de control de malezas en la productividad y calidad del pasto estrella de áfrica (Cynodon plectostachyus (K. Schum.) Pilg.). Tropical and Subtropical Agroecosystems 10: 393-404.

Fernández, M. L., J. P. Ramírez, C. Chaves \& M. Arias. (2008). Disminución en la incidencia de mastitis en ganado vacuno con la aplicación de un sellador de barrera experimental. Agronomía costarricense. Revista de ciencias agrícolas 32: 107-112.

García-Martínez, A., Albarrán-Portillo, B \& Avilés-Nova, F. (2015). Dinámicas y tendencias de la ganadería doble propósito en el sur del Estado de México. Agrociencia 49: 125-139.

Hair, F. J., B. Black, B. Babin, R. A. Anderson \& R. L. Tatham. (2006). Multivariate data analysis. (6th Ed) Upper Saddle River, New Jersey: Pearson Prentice Hall Publishing.

Hernández, G. A., H. P. A. Martínez, E. J. Zaragoza, H. H. Vaquera, G. F. Osnaya, T. M. Joaquín \& Z. M. E. Velazco. (2012). Caracterización del rendimiento de forraje de una pradera de alfalfa-ovillo al variar la frecuencia e intensidad del pastoreo. Revista fitotecnia mexicana. 35: 259-266.

Houdijk, J. G. M., I. Kyriazakis \& S. Athanasiadou. (2012). Manipulating small ruminant parasite epidemiology through the combination of nutritional strategies. Veterinary Parasitology 186: $38-50$.

Johnson, R. \& D. Wichern. (1998). Applied multivariate statistical analysis. (4th Ed). Prentice Hall, New York, USA.

Mariscal, P. C. A \& J. R. A. Moreno. (2013). Prevalencia de Haematobia irritans (Linnaeus 1758) (Díptera: Muscidae) en Bovinos de la Provincia Cercado, Beni. Revista Científica Agro ciencias Amazonias 1:31-42.

Montiel, F., C. S. Galina, C. Lamothe \& O. Castañeda. (2007). Effect of a feed supplementation during the mid-lactating period on body condition, milk yield, metabolic profile and pregnancy rate of grazing dual-purpose cows in the Mexican humid tropic. Archivos de Medicina Veterinaria 39:207-213.

Ortega-Jiménez, E., M. E. Nava-Tablada, S. Vargas-López, F. López, A. Villa-Herrera, S. LópezOrtiz. (2009). Utilización del guácimo (Guazuma ulmifolia Lam.) Como fuente de forraje en 
Relación entre las prácticas tecnológicas de manejo, la producción y su asociación con las épocas del año en el sistema de doble propósito del trópico mexicano

la ganadería bovina extensiva del trópico mexicano. Tropical and Subtropical Agroecosystems 10: 253-261.

Oviedo, C., A. Pastrana, L. Maza, R. Salgado \& O. Vergara. (2011). Supplementation of lactating calves dual purpose in the dry season in the middle Sinu valley, Colombia. Revista U.D.C.A Actualidad \& Divulgación Científica 14: 57-62.

Pinzón, T.A., M. Vásquez, F. Camilo \& M. G. Rodríguez. (2009). Subclinical mastitis effects in some dairy herds in the Upper Chicamocha River (Boyacá Department). Revista de Medicina Veterinaria 17: 23-35.

Pulido, R. G., A. Escobar, S. Follert, M. Leiva, P. Orellana, F. Wittwer \& O. Balocchi. (2009). Efecto del nivel de suplementación con concentrado sobre la respuesta productiva en vacas lecheras a pastoreo primaveral con alta disponibilidad de pradera. Archivos de medicina veterinaria 41: 197-204.

Rojo-Rubio, R., J. F. Vázquez-Armijo, P. Pérez-Hernández, G. D. Mendoza-Martínez, A. Z. M. Salem, B. Albarrán-Portillo, A. González-Reyna, J. Hernández-Martínez, S. Rebollar, D. Cardoso-Jiménez, E. J. Dorantes-Coronado \& J. G. Gutiérrez-Cedillo. (2009). Dual-purpose cattle production in Mexico. Trop. Anim. Health Prod 41: 715-721.

Sánchez, T., L. Lamela \& O. López. (2010). Efecto de la suplementación con residuos de destilería del maíz en el comportamiento de novillas en una asociación de gramínea y leucaena. Pastos y Forrajes 33: 1-8.

SAS (Statistical Analysis System). (2013). User's Guide (Release 9.4). Cary, North Carolina, USA: SAS Institute.

SIAP. (Servicio de Información Agroalimentaria y Pesquera). (2013). Población Ganadera, avícola y apícola. Resumen 2001-2014. http://infosiap.siap.gob.mx/repoAvance_siap_gb/pecResumen.jsp (Consulta: febrero 2017).

Torres-Acosta, J.F.J., D. E. Jacobs, A. J. Aguilar-Caballero, C. Sandoval-Castro, L. Cob-Galera \& M. May-Martínez. (2006). Improving resilience against natural gastrointestinal nematode infections in browsing kids during the dry season in tropical Mexico. Veterinary Parasitology 135: 163-173.

Trujillo, C. M., A. F. Gallego, N. Ramírez \& L. G. Palacio. (2011). Prevalencia de mastitis en siete hatos lecheros del oriente antioqueño. Revista Colombiana de Ciencias Pecuarias 24: 11-18. 
Vazquez, O. P \& T. R. Smith. (2000). Factors affecting pasture intake and total dry matter intake in grazing dairy cows. J. Dairy Sci 83, 2301-2309.

Waard, D \& H. Jacobus. (2010). ¿Ordeñando micobacterias del ganado? Impacto económico y en salud de Tuberculosis bovina y Paratuberculosis en Colombia. Revista MVZ Córdoba 15: $2037-2040$. 